\title{
Pinning the tail on the substrate
}

Two high-throughput 'kinomic' analysis strategies could provide kinase researchers with a bounty of information about the targets and the regulation of their favorite enzymes.

It's virtually impossible to pick up a biological research journal these days without finding papers examining different protein phosphorylation pathways. This is hardly surprising, as phosphorylation has a role in virtually every important cellular process. What may be surprising, however, is how little is actually known about the organization of this kinase-substrate 'kinome'. "There are probably thousands, if not tens of thousands, of phosphorylations in yeast-and for sure tens of thousands in humans," says Yale researcher Michael Snyder. "[But] when you actually look at what's in the literature, there [are] less than 160 known for yeast."

In a new article in Nature, Snyder's group describes an approach to resolve this problem-a high-throughput assay for the identification of proteomic targets, which they used to screen 87 different yeast kinases (Ptacek et al., 2005). The group worked with a proteomic expression library consisting of roughly $75 \%$ of the yeast proteome, spotting the proteins on duplicate arrays; they then exposed these arrays to kinases in the presence of radioactive ATP, so that specific interactions could be identified at spots that reproducibly generated a signal. Snyder's group identified over 4,000 phosphorylation events, which they assembled to create a detailed kinomic map. The assay also yielded important details about regulatory 'modules' - recurring patterns by which sets of kinases and targets interact and modulate each other's activity. "It goes to show that there are some fundamental circuits that eukaryotes just like to use," says Snyder.

Harvard researcher Erin O'Shea was also interested in developing new systems for kinase substrate identification, but rather than tackling the entire kinome, she sought information about a specific yeast cyclin-dependent kinase, Pho85. She used the 'gatekeeper' strategy first developed by Kevan Shokat's group at University of California, San Francisco, using a mutant Pho85 variant capable of catalyzing phos- phorylation with ATP analogs (Dephoure et al., 2005). O'Shea's team screened this mutant against a tagged library of over 4,000 proteins, with reactions performed in yeast extracts in an effort to replicate natural conditions. Then they immunopurified the tagged proteins; when a tagged protein was successfully labeled by the radioactive ATP analog, it was deemed a putative substrate. O'Shea's team identified 24 potential targets, several of which seemed quite feasible based on existing data. "The biological functions of the substrates we identified coincide well with the functions that have been associated with Pho85... through genetic studies," she says. "It's an encouraging sign that the substrates we identified are indeed relevant for in vivo substrates."

O'Shea's team was also surprised by the variable specificity observed with different Pho85-cyclin combinations. "The specificity exhibited by the kinase in vitro is exquisite," she says, "and we compared the ability of...two kinases that share the same catalytic subunit to phosphorylate the same substrates, and found that they have markedly distinct profiles." Snyder was similarly surprised by his group's results with the closely related Tpk kinases: "Genetically, the Tpks are thought, at least in some respects, to be functionally redundant-but we show that they're functionally distinct."

In vivo confirmation is the next key step, and O'Shea believes that such validation could provide strong support for her assay as a robust method for the detailed analysis of individual kinases. For his part, Snyder hopes his group's work will form the beginnings for an eventual atlas of the yeast kinome-with public participation being a key component. "That's why we put the data set out there for the community as soon as possible," he says. "There's a lot of work to do ahead, for sure-I think this is meant to really get things going."

\section{Michael Eisenstein}

\section{RESEARCH PAPERS}

Ptacek, J. et al. Global analysis of protein

phosphorylation in yeast. Nature 438, 679-684 (2005).

Dephoure, N. et al. Combining chemical genetics and proteomics to identify protein kinase substrates. Proc. Natl. Acad. Sci. USA 102, 17940-17945 (2005). 